Open Access

\title{
Validation of the physiological background correction method for the suppression of the spill-in effect near highly radioactive regions in positron emission tomography
}

Mercy I. Akerele ${ }^{1}$, Palak Wadhwa ${ }^{1,2}$, Jesus Silva-Rodriguez ${ }^{3}$, William Hallett ${ }^{2}$ and Charalampos Tsoumpas $^{1,2^{*}}$ (1)

\section{* Correspondence:}

c.tsoumpas@leeds.ac.uk

'Biomedical Imaging Science Department, School of Medicine, University of Leeds, Leeds, West Yorkshire, UK

${ }^{2}$ Invicro, Hammersmith Hospital, London, UK

Full list of author information is available at the end of the article

\begin{abstract}
Background: Positron emission tomography (PET) imaging has a wide applicability in oncology, cardiology and neurology. However, a major drawback when imaging very active regions such as the bladder is the spill-in effect, leading to inaccurate quantification and obscured visualisation of nearby lesions. Therefore, this study aims at investigating and correcting for the spill-in effect from high-activity regions to the surroundings as a function of activity in the hot region, lesion size and location, system resolution and application of post-filtering using a recently proposed background correction technique. This study involves analytical simulations for the digital XCAT2 phantom and validation acquiring NEMA phantom and patient data with the GE Signa PET/MR scanner. Reconstructions were done using the ordered subset expectation maximisation (OSEM) algorithm. Dedicated point-spread function (OSEM+PSF) and a recently proposed background correction (OSEM+PSF+BC) were incorporated into the reconstruction for spill-in correction. The standardised uptake values (SUV) were compared for all reconstruction algorithms.

(Continued on next page)
\end{abstract}


(Continued from previous page)

Results: The simulation study revealed that lesions within 15-20 mm from the hot region were predominantly affected by the spill-in effect, leading to an increased bias and impaired lesion visualisation within the region. For OSEM, lesion SUV $V_{\max }$ converged to the true value at low bladder activity, but as activity increased, there was an overestimation as much as 19\% for proximal lesions (distance around 15-20 mm from the bladder edge) and 2-4\% for distant lesions (distance larger than $20 \mathrm{~mm}$ from the bladder edge). As bladder SUV increases, the \% SUV change for proximal lesions is about $31 \%$ and $6 \%$ for SUV max and SUV mean, respectively, showing that the spill-in effect

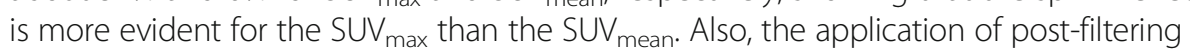
resulted in up to $65 \%$ increment in the spill-in effect around the bladder edges. For proximal lesions, PSF has no major improvement over OSEM because of the spill-in effect, coupled with the blurring effect by post-filtering. Within two voxels around the bladder, the spill-in effect in OSEM is 42\% (32\%), while for OSEM+PSF, it is 31\% (19\%), with (and without) post-filtering, respectively. But with OSEM+PSF+BC, the spill-in contribution from the bladder was relatively low (below $5 \%$, either with or without post-filtering). These results were further validated using the NEMA phantom and patient data for which OSEM+PSF+BC showed about 70-80\% spill-in reduction around the bladder edges and increased contrast-to-noise ratio up to 36\% compared to OSEM and OSEM+PSF reconstructions without post-filtering.

Conclusion: The spill-in effect is dependent on the activity in the hot region, lesion size and location, as well as post-filtering; and this is more evident in $S U V_{\text {max }}$ than $S U V_{\text {mean. }}$. However, the recently proposed background correction method facilitates stability in quantification and enhances the contrast in lesions with low uptake.

Keywords: PET, Post-filtering, Reconstruction, Background correction, Lesion contrast

\section{Background}

Positron emission tomography (PET) has established applications in oncology, cardiology and neurology [1-3] using standardised uptake value (SUV) for quantification, diagnosis and post-therapeutic response prediction [4]. However, as PET involves injection of radiotracers, concerns have been raised on the effectiveness of some common tracers in imaging areas with high radiotracer uptake (hot regions) such as the brain, urinary bladder, myocardium and spine [5-7]. These concerns stem from the observation that activity from the hot regions may interfere with PET quantification and visualisation of nearby lesions, tumours and abnormalities. As a result, nearby lesions have their SUVs overestimated, and in some cases, lesions can be totally missed [8]. This effect is often referred to as the "spill-in" or "shine-through" effect [8-10].

For example, in clinical practice, the spill-in effect from the bladder to the surroundings is often addressed using techniques such as bladder voiding prior to acquisition, catheterisation, irrigation and retrograde filling [11]. However, none of these techniques has been proven to be $100 \%$ efficient or give reliable results [12-15]. They are also uncomfortable and invasive for the patient [15], whereas PET is meant to be a minimally invasive imaging technique. Due to these challenges, alternative tracers are sometimes used because of their minimal urinary excretion [16-18], but such tracers still have some limitations with regards to patient sensitivity and specificity [19]. These issues clearly suggest the need for a more practical correction technique for the spill-in of activity from hot regions to the surroundings. 
Correction of spill-in effect is essential in PET imaging for enhanced quantitative accuracy and better lesion detection [20-23]. This correction can be voxel or region of interest (ROI)-based and implemented either within or after the image reconstruction [24-26]. However, spill-in correction is commonly applied to brain imaging, with little or no studies involving imaging of the pelvic regions. Also, some correction techniques require a priori information about the lesion shape and size; hence, increasing the possibility of erroneous estimation when this information is not precisely known. They might also be computationally demanding [26].

A recent novel simulation study [27] suggested a reconstruction approach to correct for the high physiological radioactive concentration in order to address these aforementioned issues and improve lesion quantification. This method involves segmentation, forward-projection and reconstruction-based correction. However, the performance of this correction method on a state-of-the-art scanner, including accurate iterative scatter correction and point-spread-function (PSF) correction inside the reconstruction, remains to be studied. The previous work used the General Electric (GE) Advance NXi scanner with a spatial resolution of $4.8 \mathrm{~mm}$ full width at half maximum (FWHM), whereas the present work used the GE Signa PET/MR scanner with a spatial resolution of $4.3 \mathrm{~mm}$ FWHM. Also, the previous work corrected for the scattered and random events before the reconstruction, whereas this current work incorporated all corrections (attenuation, normalisation, scatter and randoms) in the reconstruction, as in the state-of-the-art reconstruction algorithms.

The aim of this study was to implement the recently proposed background correction and evaluate its performance in terms of lesions' quantification accuracy and contrast, using both simulated and real clinical PET/MR data. The performance was assessed for lesions near a background hot region, as a function of background activity, lesion-to-background distance, lesion size, spatial resolution, and degree of post-filtering.

\section{Materials and methods}

Datasets and PET/MR system

Two datasets were primarily used in this study: (i) simulated data from digital XCAT2 phantom [28] and (ii) experimental data from a NEMA IQ phantom [29]. Additionally, a patient abdominal scan was used for further validation. These datasets are shown in Fig. 1.

The experimental studies were acquired using the GE Signa PET/MR scanner at Invicro Ltd., with $25 \mathrm{~cm}$ axial field of view (FOV), $60 \mathrm{~cm}$ transaxial FOV, $70 \mathrm{~cm}$ ring diameter, $25 \times 4.0 \times 5.3$ crystal size in $\mathrm{mm}$, and energy resolution of $10.5 \%$ with an energy window $425-650 \mathrm{keV}$ [30]. The simulation was performed using an analytical model of the same scanner.

\section{Simulations}

We simulated the pelvic bed using digital XCAT2 phantom with a typical $\left[{ }^{18} \mathrm{~F}\right]$-fluorodeoxyglucose $\left(\left[{ }^{18} \mathrm{~F}\right]-\mathrm{FDG}\right)$ distribution. Hot bladder was simulated with a fixed volume $(500 \mathrm{ml})$ and various activities (i.e. SUVs 8.5, 19.3, 33.8 and 55.5) allocated as obtained from the literature [31,32]. This is to mimic the progressive increase in bladder activity due to the radiotracer accumulation during a typical PET examination. Three lesions labelled L1-L3 each with diameter $10 \mathrm{~mm}$ and fixed activity value (SUV 8) were placed 


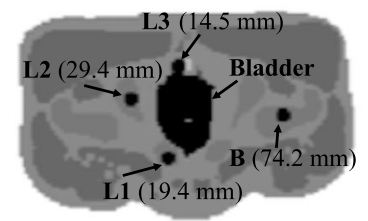

(a)

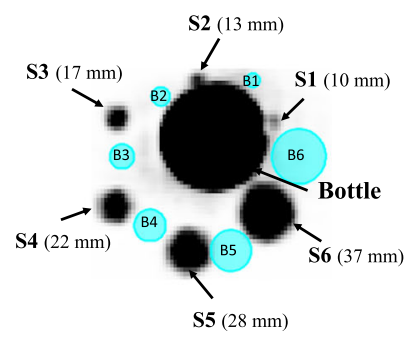

(b)

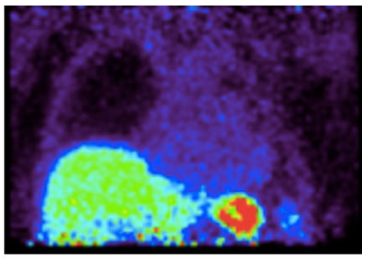

(c)

Fig. 1 The datasets used for this study: a simulation from XCAT2 phantom consisting of the bladder, three lesions (L1-L3) and one background lesion (B) placed at different distances (shown in brackets) from the bladder; $\mathbf{b}$ NEMA phantom consisting of a hot bottle at the centre, surrounded by six spheres (S1-S6) with diameters shown in brackets. The blue spheres (B1-B6) were used to estimate the background activity for the estimation of contrast-to-noise ratio (CNR); $\mathbf{c}$ patient data showing high activity in the spleen and liver

at different locations around the bladder as shown in Fig. 1a. We also simulated an increase in bladder volume and activity during a typical 90 min scan using extracted values from the literature [33, 34] at time points $1,10,30,45$ and $90 \mathrm{~min}$. This was done to investigate the spill-in effect from the bladder to the surrounding lesions as a function of increasing bladder volume, bladder activity and lesion distance from the bladder. Additionally, lesion diameters ranging from $6 \mathrm{~mm}$ to $12 \mathrm{~mm}$ with a step size of $2 \mathrm{~mm}$ were simulated for all bladder SUVs to investigate the spill-in effect as a function of lesion size. For each lesion diameter, we also added same-sized background lesion (B) farther from the bladder such that the background lesion is not affected by the spill-in effect from the bladder. This was done to distinguish between spill-in and spill-out effects as it affects lesion quantification, especially for small lesions. The emission and attenuation images were generated for each of these bladder and lesions combinations.

Fully three-dimensional (3D) analytical simulation was done using the Software for Tomographic Image Reconstruction (STIR) package [35], considering all sinograms with ring differences (RD) less than or equal to 44 , and span 1 . To approximate the blurring effect from the scanner, the simulated images were first convolved with a Gaussian filter having full width at half maximum (FWHM) as that of GE PET/MR PSF ( $4.2 \mathrm{~mm}$ and 5.7 $\mathrm{mm}$ FWHM in transverse and axial planes, respectively [36]) at the forward-projection stage. The forward projector used is based on Siddon's ray tracing algorithm [37], tracing 10 tangential rays for each bin. Attenuation correction factors were calculated from the attenuation image, and this was used to attenuate the emission sinogram. Constant normalisation and random sinograms were also generated, with the random counts making up to $20 \%$ of the total projection data. Scatter was estimated analytically using the single scatter simulation (SSS) approach and scaled to make the scatter count $35 \%$ of the total simulated events. The random and scatter sinograms were used to generate the additive term, while the attenuation and normalisation sinograms were used as multiplicative terms. Time of flight (TOF) was not simulated for this study.

Poisson noise was added to the sinograms to simulate $65 \times 10^{6}$ counts, and 20 noise realisations were performed for statistical analysis. Spherical ROIs equal in diameter to the size of the lesions were placed in the position of each lesion in order to extract the uptake values $\left(\mathrm{SUV}_{\text {mean }}\right.$ and $\left.\mathrm{SUV}_{\max }\right)$ for each noise realisation. The mean $(\bar{M})$, 
standard error of the mean $(S E M)$ and bias $(B)$ from all the 20 realisations (as defined in Eq. 1(i-iv)) were used as figures of merit to show the differences in SUV values for both uncorrected and corrected images.

$$
\begin{aligned}
& \bar{M}=\frac{1}{N} \sum_{i=1}^{N} f_{i} \\
& S E M=\frac{S D}{\sqrt{N}}
\end{aligned}
$$

where

$$
\begin{aligned}
& S D=\sqrt{\frac{1}{N-1} \sum_{i=1}^{N}\left(f_{i}-\bar{M}\right)^{2}} \\
& B=\frac{\bar{M}-T}{T} \times 100
\end{aligned}
$$

$f_{i}$ is the SUV from a single noise realisation, $N$ is the total number of noise realisations $(=20)$ and $T$ is the SUV of the true simulated image.

The \% change in lesion $(l)$ SUV $\left(\Delta S U V_{l}\right)$ as the bladder SUV increases from SUV 8.5 to SUV 55.5 was also estimated using:

$$
\Delta S U V_{l}(\%)=\frac{S U V_{l(55.5)}-S U V_{l(8.5)}}{S U V_{l(8.5)}} \times 100
$$

where $S U V_{l(8.5)}$ and $S U V_{l(55.5)}$ represent the lesion SUV at bladder SUVs 8.5 and 55.5, respectively.

To further investigate the spill-in effect from the bladder to the surroundings, we created shells of different voxels (from 2 to 10 voxels with a step size of 2 voxels) around the bladder by performing a morphological operation on the bladder mask to obtain the edge mask:

$$
\text { edge mask }=\text { dilation }(\text { bladder mask, } n) \text {-bladder mask }
$$

where dilation(bladder mask, $n$ ) means dilating the bladder mask by $n$ voxels using a sphere structuring element in MATLAB.

The resulting edge mask was then used to extract the uptake values around the bladder in both true simulated and reconstructed mean images as illustrated in Fig. 2a.

\section{Validation by real data}

\section{Experimental phantom}

A phantom experiment was performed with the GE Signa PET/MR scanner at Invicro Ltd. using the NEMA image quality (IQ) phantom without the wall (Fig. 1b) [29]. This phantom consisted of six fillable spherical spheres S1 to S6 (with diameters 10, 13, 17, 22, 28 and $37 \mathrm{~mm}$, respectively, and filled with $5.38 \mathrm{MBq}$ of $\left[{ }^{18} \mathrm{~F}\right]-\mathrm{FDG}$ ). A high-activity $500 \mathrm{~mL}$ bottle (filled with $77.9 \mathrm{MBq}$ of $\left[{ }^{18} \mathrm{~F}\right]-\mathrm{FDG}$ ) was placed at the centre of the phantom. The experimental set-up involved a simultaneous PET/MR acquisition with a 5-min static PET acquisition and 2-point Dixon MR 3D acquisition. List of events and singles rates for crystals were extracted from the listmode file from the scanner, and these were converted to emission and random sinograms using STIR utility. The normalisation file was also extracted from the scanner and was converted to the 


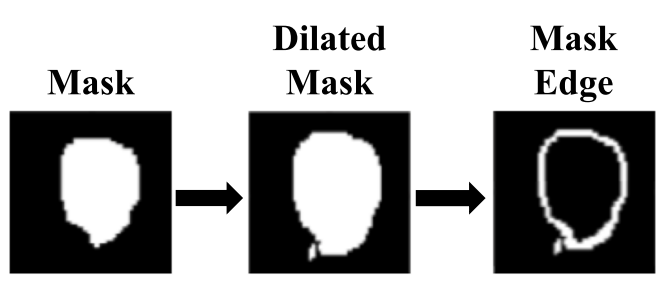

(a)

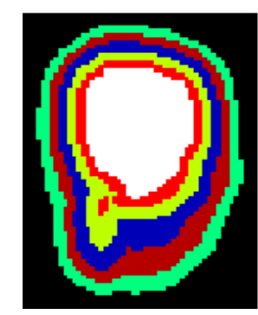

(b)

Fig. 2 The schematic 2D representation of the technique used to extract the voxel values around the edges of the simulated bladder. a represents the procedure for extracting the voxel values, while $\mathbf{b}$ shows a $2 \mathrm{D}$ representation of all the dilated $3 \mathrm{D}$ regions around the bladder (i.e. the white region) from 2 voxels to 10 voxels with a step size of 2 voxels

normalisation sinogram using STIR. Attenuation factors were obtained from the in-phase MRAC image, and scatter was estimated using STIR.

The contrast-to-noise ratio (CNR) was used to evaluate the signal quality and noise properties of each reconstruction algorithm. This was done by estimating the mean activity and standard deviation in each sphere, as well as in pre-selected background spheres. The background spheres were represented as six spherical ROIs placed around the hot bottle which were exactly of the same size as the spheres and located at approximately the same distance from the hot bottle (as shown in Fig. 1b).

So, the CNR was estimated for each sphere $(i=1,2, \ldots, 6)$ using:

$$
\mathrm{CNR}_{\text {sphere }(i)}=\frac{\operatorname{Activity}_{\text {Sphere }(i)}-\operatorname{Activity}_{\text {Background }(i)}}{\sqrt{\mathrm{SD}_{\text {Sphere }(i)}^{2}+\mathrm{SD}_{\text {Background }(i)}^{2}}}
$$

Also, the spill-in activity from the bottle to the surroundings was estimated using the same morphological operation as in the simulation studies (shown in Eq. 3).

\section{Patient data}

For further validation, we used patient data (Fig. 1c) acquired with the GE PET/MR scanner at Invicro Ltd. This was acquired during a lung fibrosis examination using an $\left[{ }^{18} \mathrm{~F}\right]$-based radiotracer. This data did not exhibit high activity in the bladder, but in the spleen and the liver. Thus, for demonstration purposes, we chose the spleen as the background hot region. We reconstructed the patient data using the same settings as for the phantom experiment. We also carried out the morphological dilation operation (in Eq. 3) on all reconstructed images so as to estimate the spill-in effect around the hot region (spleen).

\section{Data reconstruction and spill-in correction}

Image reconstruction was done using the 3D iterative ordered subset expectation maximisation (OSEM) algorithm in STIR library. Attenuation, normalisation, random and scatter corrections were performed by including the multiplicative and additive terms in the reconstruction algorithm. For the simulation, 28 subsets and 30 full iterations were used to ensure reasonable convergence of the recently proposed correction algorithm. Meanwhile, for the real data, fully 3D reconstruction with mixed span factors 
according to GE's configuration (span 1 for $|\mathrm{RD}|>1$; and span 3 for $\mathrm{RD}=-1,0,1$ ) was done using OSEM with 28 subsets and 20 full iterations, incorporating all corrections within the reconstruction. The reconstructed images have $256 \times 256 \times 89$ voxels with size $2.34 \times 2.34 \times 2.78 \mathrm{~mm}^{3}$, and they were post-filtered with a 4-mm isotropic 3D Gaussian filter.

Spill-in correction was done using the two techniques as outlined below:

\section{(a) PSF reconstruction}

This involves incorporating a spatially invariant PSF into the OSEM reconstruction in both forward and backward projections as illustrated in Eq. (5). This PSF was specified as a 3D Gaussian filter with $4.2 \mathrm{~mm}$ and $5.7 \mathrm{~mm}$ FWHM in transverse and axial planes, respectively, according to experimental values obtained for the GE PET/MR scanner [36].

$$
H_{i j}^{\prime}=\sum_{k} H_{k j} P S F_{i k}
$$

where $H_{k j}$ represents the system matrix and $H_{i j}^{\prime}$ is the system matrix convolved with the system PSF in both forward and back projections [38, 39]. This reconstruction method is referred to as OSEM+PSF in this study.

\section{(b) Background correction (BC)}

For the simulation, the bladder (hot region) was automatically segmented from the XCAT2 phantom to obtain the bladder mask $S_{j}$. This was then multiplied by the OSEM+PSF reconstructed image $f_{j}^{(N)}$ (taken at five iterations) to obtain the bladder contribution in the image (i.e. $B_{j}=S_{j} f_{j}^{(N)}$ ), which was then forward-projected using the same projection matrix for the simulation $\left(P_{i}=\sum_{j} H_{i j}^{\prime} B_{j}\right)$ and included in the reconstruction as a background term along with the additive term (as shown in Eq. 6). For the real data, the highly radioactive bottle that accompanied the NEMA phantom and the hot spleen of the patient were segmented from the Dixon in-phase MRAC image before forward-projecting it to obtain the background contribution.

$$
f_{j}^{(n+1)}=\frac{f_{j}^{(n)}}{\sum_{i \in J_{j}} H_{i j}^{\prime} M_{i}} \sum_{i \in J_{j}} H_{i j}^{\prime} \frac{y_{i} M_{i}}{M_{i} \sum_{k \in I_{i}} H_{i k}^{\prime} f_{k}^{(n)}+A_{i}+P_{i}}
$$

where $f_{j}^{(n)}$ is the uncorrected image, $y_{i}$ is the emission sinogram, $H_{i j}^{\prime}$ is the system matrix with the PSF, $M_{i}$ is the multiplicative term, $A_{i}$ is the additive term, $P_{i}$ is the bladder background term and $f_{j}^{(n+1)}$ is the final corrected image without the bladder contribution. The flow sequence is shown in Fig. 3. Since this technique also includes OSEM $+\mathrm{PSF}$, it will be referred to as $\mathrm{OSEM}+\mathrm{PSF}+\mathrm{BC}$ in this study.

\section{Results}

\section{Simulations}

Investigating the spill-in effect as a function of bladder activity, lesion size and post-filter using OSEM reconstruction

Figure 4 shows the mean lesion $\mathrm{SUV}_{\text {max }}$ and $\mathrm{SUV}_{\text {mean }}$ for OSEM reconstruction at 30 full iterations. As the bladder SUV increases, the lesion $\mathrm{SUV}_{\max }$ also increases for all 


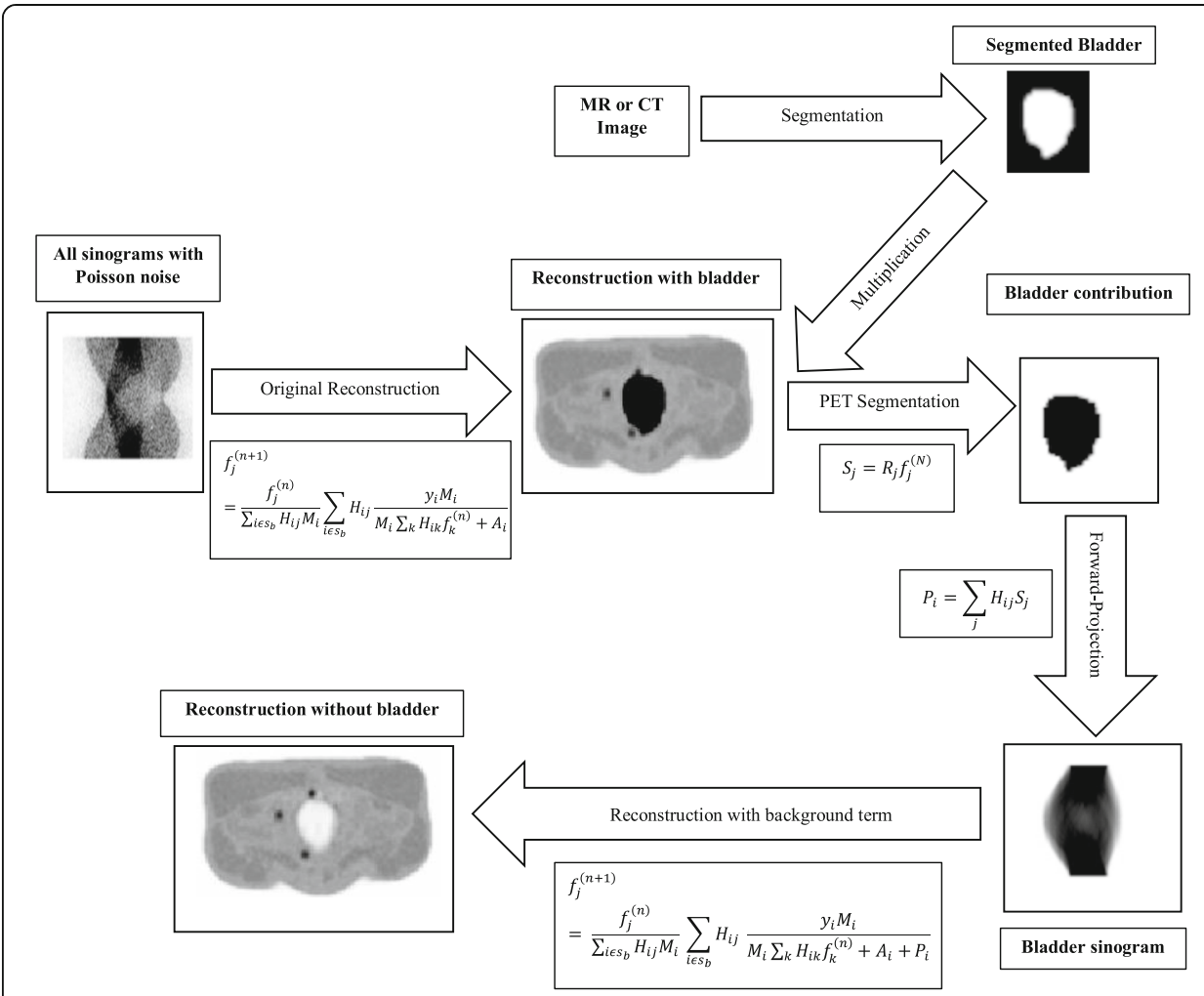

Fig. 3 Schematic description of the background correction technique, as demonstrated for the XCAT2 phantom simulation. In this study, the bladder was automatically segmented from the XCAT2 phantom, but in principle, the hot region can be segmented from either the CT or MR image

lesions, with the highest SUV in L3. However, SUV mean increases only for L3. The \% bias and the \% change in lesion SUV as the bladder SUV increases from SUV 8.5 to SUV 55.5 (using Eq. 2) are presented in Table 1.

For lesion L3, the \% bias in SUV increases as the bladder SUV increases, and this is more pronounced for $\mathrm{SUV}_{\max }$ than $\mathrm{SUV}_{\text {mean. }}$. However, for lesions L1 and L2, only
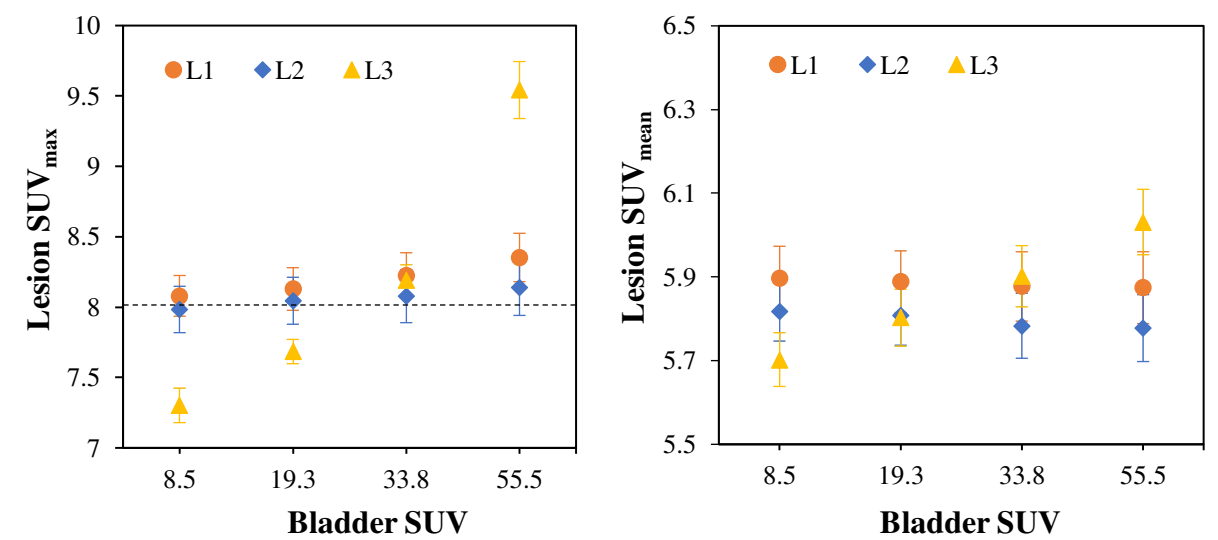

Fig. 4 The lesion uptake as the bladder SUV increases for images reconstructed with OSEM. These are the mean SUVs from all the 20 noise realisations and for lesion diameter $10 \mathrm{~mm}$ at 30 full iterations. The error bars are the standard error of the mean (SEM) while the dashed horizontal line denotes the true simulated lesion SUV 
Table 1 The bias and relative change in lesion SUV max and SUV $V_{\text {mean }}$ for all the simulated bladder SUVS and for lesion diameter $10 \mathrm{~mm}$. These are estimated from the mean SUVS from all the noise realisations. (The \% bias values are given in parentheses)

\begin{tabular}{|c|c|c|c|c|c|c|}
\hline \multirow{3}{*}{$\begin{array}{l}\text { Bladder } \\
\text { SUV }\end{array}$} & \multicolumn{6}{|c|}{ Simulated lesions } \\
\hline & \multicolumn{2}{|l|}{ L1 } & \multicolumn{2}{|l|}{ L2 } & \multicolumn{2}{|l|}{ L3 } \\
\hline & $S U V_{\max }$ & SUV $V_{\text {mean }}$ & $S U V_{\max }$ & $S U V_{\text {mean }}$ & $S U V_{\max }$ & $S U V_{\text {mean }}$ \\
\hline SUV 8.5 & $8.08(1.00)$ & $5.90(-26.28)$ & $7.98(-0.21)$ & $5.82(-27.27)$ & $7.30(-8.72)$ & $5.70(-28.72)$ \\
\hline SUV 19.3 & $8.13(1.61)$ & $5.89(-26.39)$ & $8.05(0.57)$ & $5.81(-27.39)$ & $7.68(-3.95)$ & $5.80(-27.47)$ \\
\hline SUV 33.8 & $8.23(2.82)$ & $5.88(-26.53)$ & $8.08(0.97)$ & $5.78(-27.71)$ & $8.19(2.41)$ & $5.90(-26.23)$ \\
\hline SUV 55.5 & $8.35(4.41)$ & $5.87(-26.57)$ & 8.14 (1.78) & $5.78(-27.78)$ & $9.54(19.27)$ & $6.03(-24.61)$ \\
\hline$\%$ Change & 3.37 & -0.39 & 1.99 & -0.70 & 30.67 & 5.77 \\
\hline
\end{tabular}

$\mathrm{SUV}_{\max }$ has an increased \% bias as the bladder SUV increases, $\mathrm{SUV}_{\text {mean }}$ seems constant. At bladder SUV 55.5, SUV $\max$ of lesion L3 (closest to the bladder) has a \% bias of 19.3\% while for L2 (further away), the \% bias in $\mathrm{SUV}_{\max }$ is only $1.8 \%$. As bladder SUV increases from 8.5 to 55.5 , the \% SUV change for L3 is $30.67 \%$ and $5.77 \%$ for $\mathrm{SUV}_{\max }$ and $\mathrm{SUV}_{\text {mean }}$, respectively, while the \% change in $\mathrm{SUV}_{\max }$ is less than $4 \%$ for lesions L1 and L2.

Figure 5 shows the spill-in and spill-out effects on lesion quantification as a function of lesion size. This is shown for lesions L1-L3 and background lesion B at bladder SUV 55.5. As expected, the spill-out effect from the lesions to the colder background causes an underestimation in lesion SUV for small diameter lesions, but the SUV increases as the diameter increases. However, the lesion SUV is further influenced by spill-in effect from the bladder to the lesions, and this depends on the lesion distance. Lesion L3, which is the closest lesion to the bladder, has the highest SUVs while lesion L2, which is farther away, has the least.
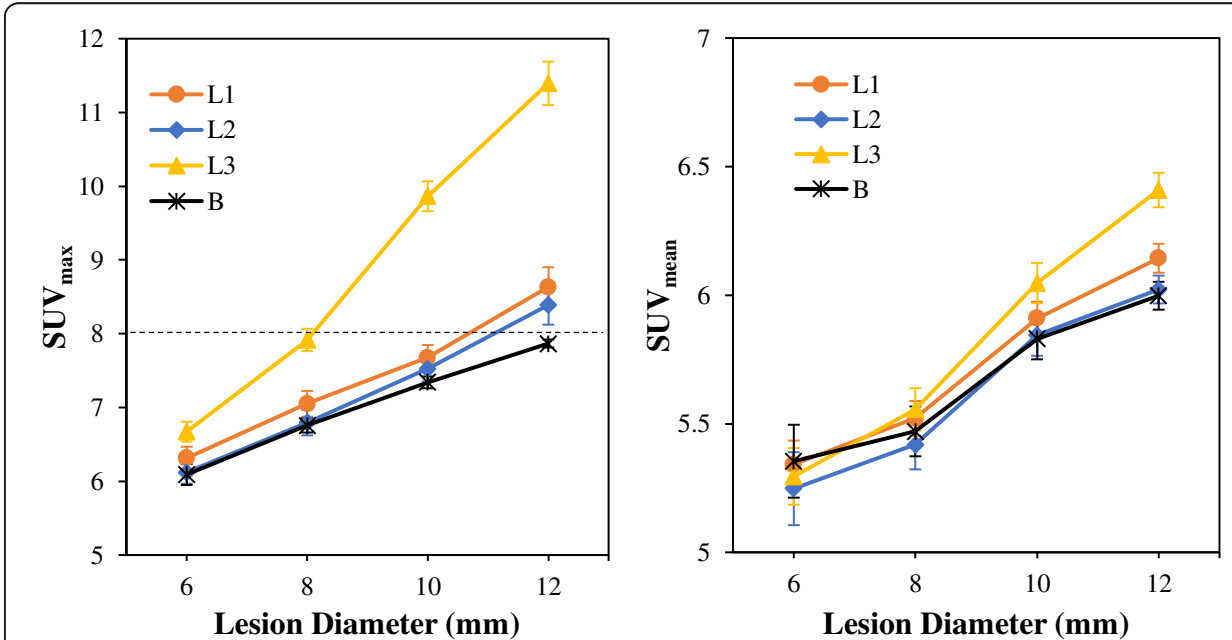

Fig. 5 The spill-in and spill-out effects as a function of lesion size for lesions L1-L3 and background lesion B at bladder SUV 55.5. The SUVS were obtained from OSEM reconstructed images using the mean SUVS of all noise realisations at 30 full iterations with a 4-mm Gaussian post-filter. The error bars are the standard error of the mean (SEM) while the dashed horizontal line denotes the true simulated lesion SUV. Background lesion B is the reference which shows the expected lesion SUV without spill-in effect 


\section{Effect of correction techniques on reduction of spill-in effect}

Figure 6 shows how the lesion SUV changes iteratively for all reconstruction techniques as bladder SUV increases from SUV 8.5 to SUV 55.5. This shows that \% change in lesion SUV reduces as iteration increases. At 30 iterations, the \% change in lesion $\mathrm{SUV}_{\max }$ is about $31 \%, 26 \%$ and $4 \%$ for OSEM, OSEM+PSF and OSEM+PSF+BC, respectively, while for lesion $\mathrm{SUV}_{\text {mean }}$, it is about $6 \%, 1 \%$ and $-6 \%$ for OSEM, OSEM+PSF and OSEM+PSF $+\mathrm{BC}$, respectively.

Table 2 shows the estimated spill-in activity from the bladder to the surrounding regions for both uncorrected and corrected images at 30 full iterations with and without post-filtering. This is the percentage activity difference in the dilated shells between the true simulated image and the reconstructed image. There is a large spill-in activity within 2 voxels around the bladder as the \% activity difference between the simulated and reconstructed images is $31.5 \%$ (41.8\%) for OSEM and $18.7 \%$ (30.8\%) for OSEM + PSF reconstructions without and with post-filtering, respectively. This shows that filtered images show increased spill-in activity of about 33\% and 65\% for OSEM and OSEM+PSF respectively, compared with the unfiltered images. At 4 voxels around the bladder, the activity difference around the bladder dropped greatly to $9.3 \%(8.5 \%)$ and $2.6 \%(2.7 \%)$, and at 10 voxels from the bladder, the activity difference is $2.8 \%$ (3.4\%) and $0.6 \%(1.5 \%)$ for OSEM and OSEM+PSF, respectively. However, with the correction technique, the \% activity difference is within $0.5 \%$ and $4.7 \%$ with and without post-filtering. It could be observed that OSEM has the highest upper limit of agreement (LOA), compared to OSEM+PSF and OSEM+PSF+BC. Also, the upper LOA for all methods reduces as we move voxels away from the bladder.

This spill-in activity can also be seen around the bladder edges in the uncorrected images (as shown in Fig. 7). This causes a bias around the bladder edges making the bladder appear bigger, hence affecting the visibility of nearby lesions.
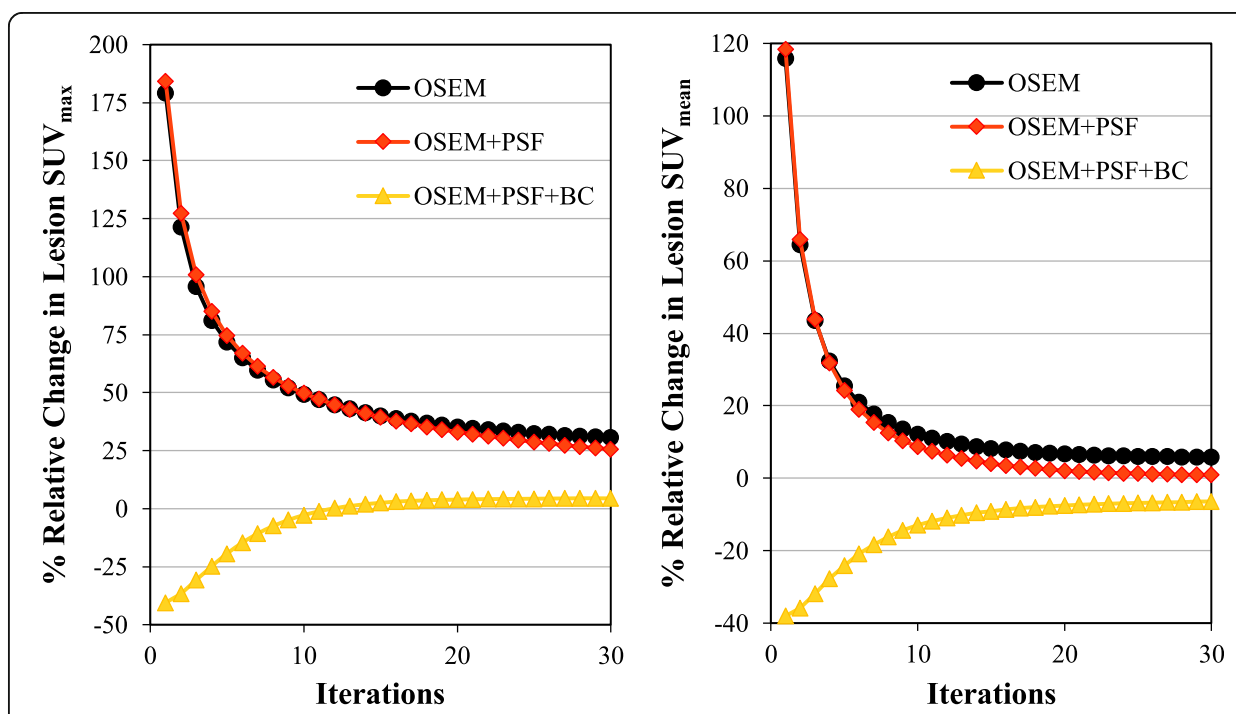

Fig. 6 The \% relative change in lesion SUV (as bladder SUV increases from SUV 8.5 to SUV 55.5) as a function of increasing iteration. This is obtained from a single noise realisation and for lesion $\mathrm{L} 3$ of diameter $10 \mathrm{~mm}$ with a 4-mm Gaussian post-filter 
Table 2 Estimation of the spill-in activity from the bladder to the surrounding regions. This is estimated from both filtered and unfiltered mean images with simulated bladder SUV 55.5 at 30 iterations (the filtered results are given in parentheses). $(\bar{d})$ is the difference between the mean values of the true simulated and reconstructed images for all voxels in the dilated region. LOA is the $95 \%$ Limit of Agreement of $(\bar{d})$, with \pm showing the upper and lower limits

\begin{tabular}{|c|c|c|c|c|}
\hline Dilated regions (voxels) & & OSEM & OSEM+PSF & $\mathrm{OSEM}+\mathrm{PSF}+\mathrm{BC}$ \\
\hline \multirow[t]{3}{*}{$1-2$} & \% Difference $(\bar{d})$ & $31.5(41.8)$ & $18.7(30.8)$ & $3.3(1.6)$ \\
\hline & $\mathrm{SD}^{1}$ & $26.4(28.4)$ & $25.0(30.2)$ & $15.4(11.7)$ \\
\hline & $\mathrm{LOA}^{2}$ & $\begin{array}{l}-20.3 \text { to }+83.3 \\
(-13.9 \text { to }+97.6)\end{array}$ & $\begin{array}{l}-30.4 \text { to }+67.8 \\
(-28.4 \text { to }+89.9)\end{array}$ & $\begin{array}{l}-26.9 \text { to }+33.5 \\
(-21.4 \text { to }+24.6)\end{array}$ \\
\hline \multirow[t]{3}{*}{$3-4$} & \% Difference $(\bar{d})$ & $9.3(8.5)$ & $2.6(2.7)$ & $4.7(3.9)$ \\
\hline & SD & $10.6(9.5)$ & $8.0(8.9)$ & $10.0(8.8)$ \\
\hline & LOA & $\begin{array}{l}-11.4 \text { to }+30.0 \\
(-10.1 \text { to }+27.0)\end{array}$ & $\begin{array}{l}-13.1 \text { to }+18.4 \\
(-14.7 \text { to }+20.1)\end{array}$ & $\begin{array}{l}-15.0 \text { to }+24.3 \\
(-13.4 \text { to }+21.2)\end{array}$ \\
\hline \multirow[t]{3}{*}{$5-6$} & $\%$ Difference $(\bar{d})$ & $5.4(6.4)$ & $2.4(3.6)$ & $1.3(2.5)$ \\
\hline & SD & $12.9(7.8)$ & $8.7(6.7)$ & $10.9(8.3)$ \\
\hline & LOA & $\begin{array}{l}-19.9 \text { to }+30.7 \\
(-8.8 \text { to }+21.6)\end{array}$ & $\begin{array}{l}-14.8 \text { to }+19.5 \\
(-9.5 \text { to }+16.7)\end{array}$ & $\begin{array}{l}-20.0 \text { to }+22.7 \\
(-13.8 \text { to }+18.8)\end{array}$ \\
\hline \multirow[t]{3}{*}{$7-8$} & \% Difference $(\bar{d})$ & $4.0(4.3)$ & $1.6(2.0)$ & $1.4(1.6)$ \\
\hline & SD & $12.8(7.9)$ & $8.9(7.1)$ & $11.2(8.3)$ \\
\hline & LOA & $\begin{array}{l}-21.1 \text { to }+29.9 \\
(-11.2 \text { to }+19.8)\end{array}$ & $\begin{array}{l}-16.0 \text { to }+19.1 \\
(-12.0 \text { to }+15.9)\end{array}$ & $\begin{array}{l}-20.4 \text { to }+23.3 \\
(-14.6 \text { to }+17.9)\end{array}$ \\
\hline \multirow[t]{3}{*}{ 9-10 } & \% Difference $(\bar{d})$ & $2.8(3.4)$ & $0.6(1.5)$ & $0.5(1.3)$ \\
\hline & SD & $11.6(7.0)$ & $9.1(6.4)$ & $10.7(7.4)$ \\
\hline & LOA & $\begin{array}{l}-19.9 \text { to }+25.5 \\
(-10.2 \text { to }+17.1)\end{array}$ & $\begin{array}{l}-17.2 \text { to }+18.4 \\
(-11.0 \text { to }+14.1)\end{array}$ & $\begin{array}{l}-20.4 \text { to }+21.3 \\
(-13.1 \text { to }+15.8)\end{array}$ \\
\hline
\end{tabular}

${ }^{1} S D=\sqrt{\frac{1}{n-1} \sum_{k=1}^{n}\left(d_{k}-\bar{d}\right)^{2}}, k$ represents each voxel in the dilated region ${ }^{2} L O A=\bar{d} \pm 1.96 S D$

\section{Dependence of the background correction method on segmentation accuracy}

To demonstrate the dependence of the recently proposed background correction method on segmentation accuracy, we simulated an increase in bladder volume and activity during a 90-min scan typical of PET/MR scan at time points 1, 10, 30, 45 and 90 min. Each image was reconstructed with background correction (OSEM+PSF+BC), but the segmented bladder mask at 1 min timepoint was used for the correction in all cases. The resulting background corrected images are displayed in Fig. 8.

This result shows that bladder expands majorly at the anterior superior end towards the rectum and the prostate. The error in segmentation is pronounced after 10 mins.

\section{Validation by phantom experiment and patient data}

Figure 9 shows the MRAC, segmented bottle and reconstructed images of the bottle phantom. OSEM+PSF+BC image shows improved visibility especially for S1 and S2.

Figure 10 shows the CNR of all the spheres as a function of iteration. As expected, the CNR increases as the sphere diameter increases, but reduces with the number of iterations. OSEM and OSEM+PSF have almost the same CNR for all spheres, while $\mathrm{OSEM}+\mathrm{PSF}+\mathrm{BC}$ shows higher CNR than OSEM and OSEM+PSF for the two smallest spheres, and the ones closest to the bottle (S1 and S2). OSEM+PSF+BC does not show any clear improvement over OSEM and OSEM+PSF for larger spheres (S3-S6). 


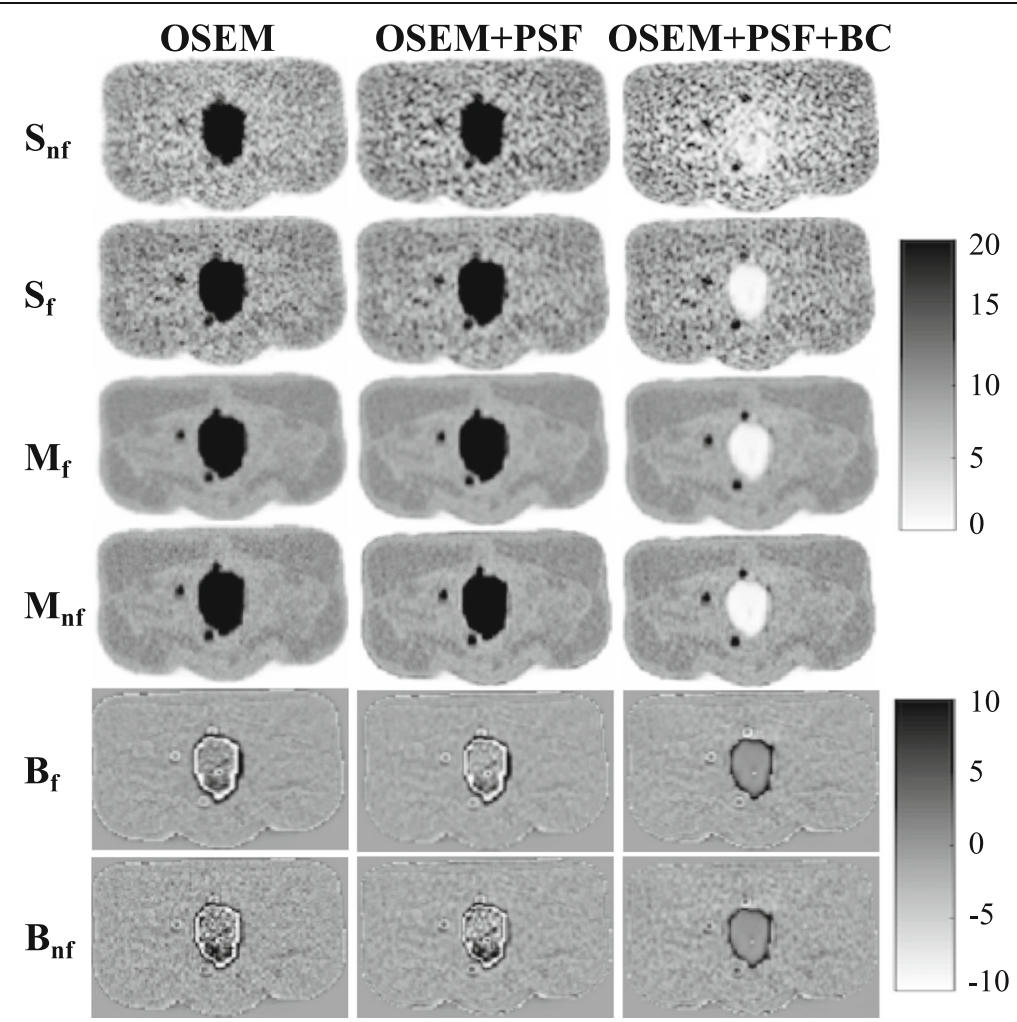

Fig. 7 Images showing improvement in lesion detection and reduction of bias around bladder edges with the correction technique. This is shown for bladder SUV 55.5 and lesion diameter $10 \mathrm{~mm}$ at 20 full iterations. $\mathrm{S}_{\mathrm{f}}$ and $S_{n f}$ are the single noise realisation images with and without filtering, respectively, while $M_{\mathrm{f}}$ and $M_{\mathrm{nf}}$ are the mean images from 20 noise realisations with and without filtering respectively. $B_{f}$ and $B_{n f}$ are the bias images (i.e. difference between the mean image and the true simulated image) with and without filtering respectively. The bias image in OSEM+PSF+BC was estimated by first removing the bladder from the true simulated image

Figure 11 shows the normalised mean activity in each of the dilated shell surrounding the hot NEMA bottle. Although the surrounding shells should have zero activity, the reconstructed images show a considerable amount of spill-in activity from the hot bottle to the surrounding shells. Within 1 voxel around the hot region, the activity is about $75 \%$ and $90 \%$ of the activity in the sphere for OSEM; and $60 \%$ and $80 \%$ for OSEM+PSF, with and without post-filtering respectively. However, at 4 voxels, this activity is greatly reduced to just about $2 \%$ for OSEM and OSEM+PSF, either with or without post-filtering. However, for OSEM+PSF+BC, the activity is less than $3 \%$ in all cases, showing an improvement of more than $80 \%$ and $70 \%$ over OSEM and OSEM+PSF reconstructions respectively.

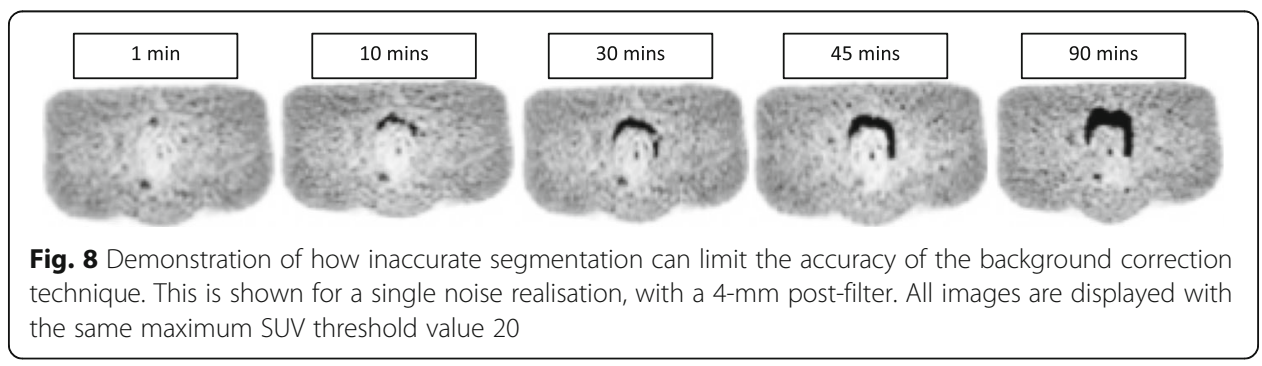



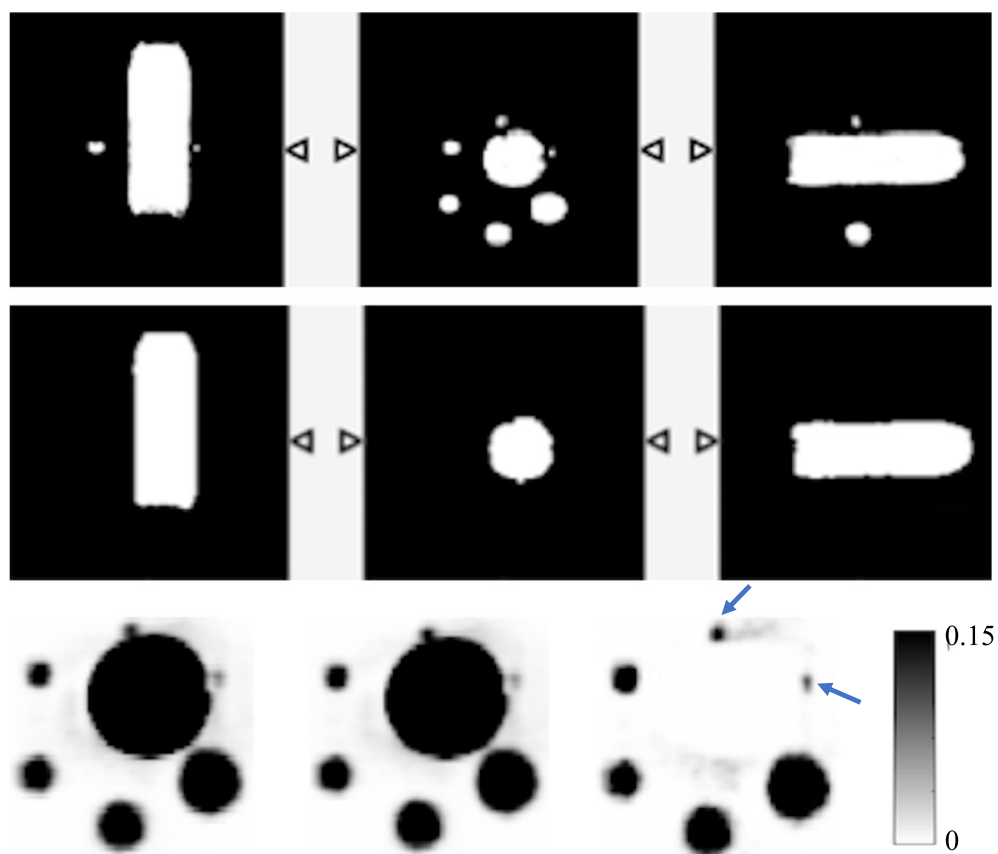

Fig. 9 The NEMA bottle phantom used for the validation. The first row shows the MRAC image of the phantom and the middle row is the segmented bottle from the MRAC image, while the last row is showing the coronal view of the reconstructed images at three full iterations with a 4-mm Gaussian filter for OSEM, OSEM+PSF and OSEM+PSF+BC reconstructions, respectively. The blue arrows in the reconstructed images are pointing to the spheres in which there is visual improvement
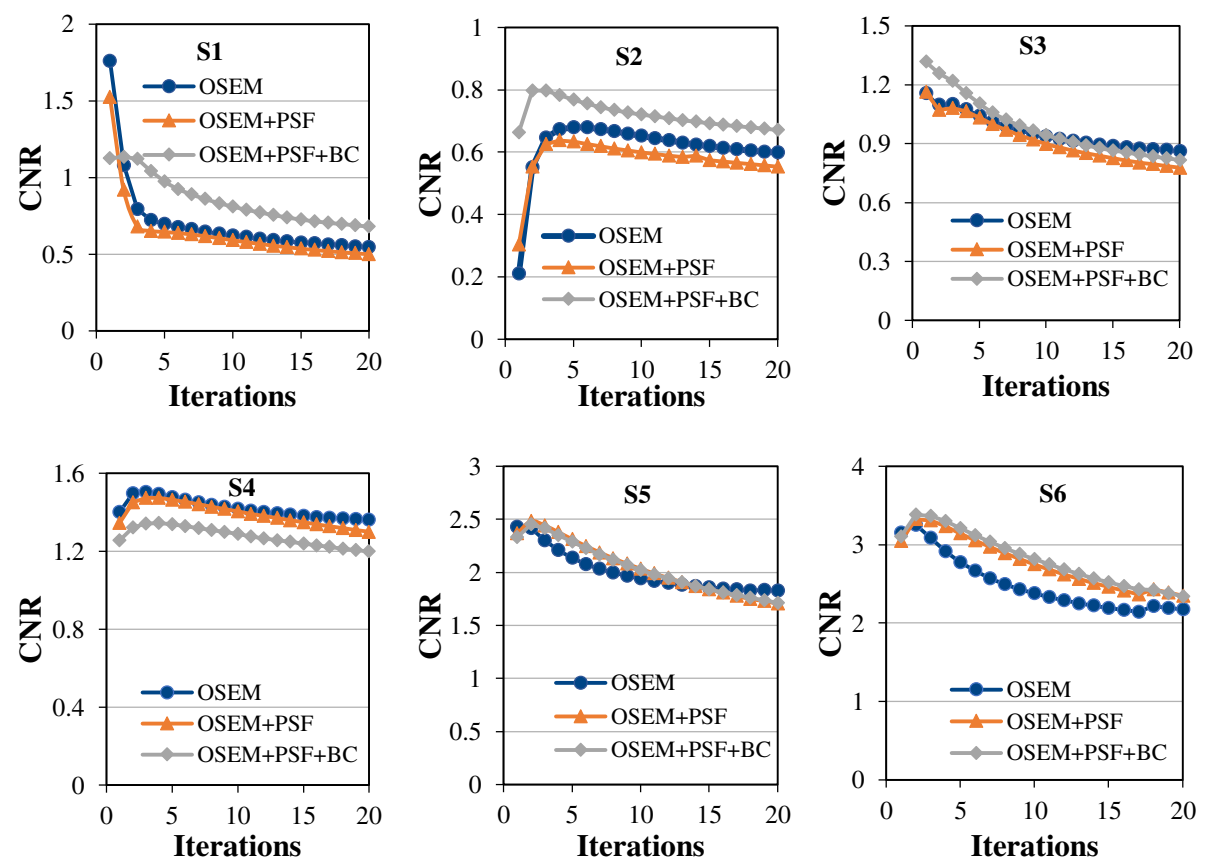

Fig. 10 Plots of the CNR of the NEMA phantom spheres S1-S6 against the iteration for all the reconstruction techniques across the 20 full iterations and without post-filtering. The CNR was estimated using the mean activity values in the spheres and background 


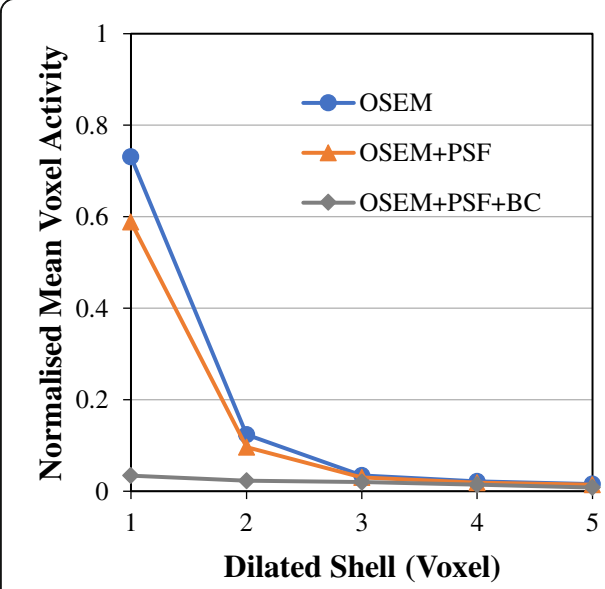

(a)

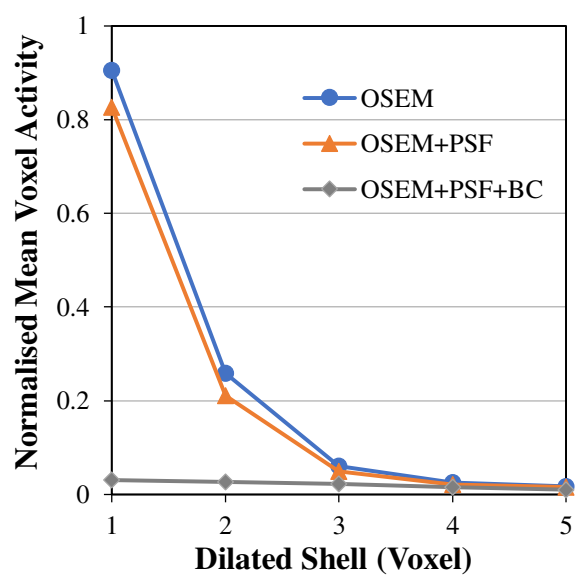

(b)

Fig. 11 The normalised mean activities within the dilated shells surrounding the NEMA bottle obtained at three full iterations with $\mathbf{a}$ no post-filter and $\mathbf{b} 4-\mathrm{mm}$ Gaussian post-filter. This demonstrates how spill-in activity from the hot bottle to the surrounding shells reduces as we move farther away from the bottle. The voxel activities were normalised with the actual activity in the spheres

For the patient data, Fig. 12 shows the normalised mean activity in the dilated shells around the spleen in all reconstructed images at 3 and 20 full iterations with and without post-filter. As expected, the mean activity in the dilated shells reduces as we move voxels away from the spleen, with the highest value at 1 voxel from the spleen in all cases. Also, increasing the number of iterations tends to reduce the activity in the dilated shell. For OSEM at voxel 1 and without post-filter, the mean activity is about 43\% at three iterations but reduced to only $26 \%$ at iteration 20 . However, with the application of post-filter, there is no pronounced difference in the voxel activities at 3 and 20 iterations. Also, OSEM+PSF has similar or even higher voxel activity than OSEM at three iterations with and without post-filter, but at 20 iterations, OSEM+PSF shows reduced voxel activity than OSEM. But in all cases, OSEM+PSF+BC has lower and almost constant voxel activity.

\section{Discussion}

We have carried out an extensive investigation with simulated and experimental phantoms on the full suppression of the spill-in effect from background hot regions to the surrounding lesions as a function of increasing background activity, lesion size, and distance to the background, using a recently proposed background correction technique [27].

The simulation results show that lesion uptake increases as bladder activity increases (Fig. 4), and this could be attributed to bladder accumulation, thereby causing spill-in effects [8]. L3 closest to the bladder has the highest change in SUV, while L1 and L2 further away have less SUV variation. This indicates that there is a high probability of SUV overestimation in lesions close to the bladder, and hence, they suffer greatly from the spill-in effect $[33,40]$. A similar experiment was conducted by Liu [8] and he concluded that if the lesions are within $40-50 \mathrm{~mm}$ from the hot source, the estimated SUV values are overestimated and therefore invalid. However, for our study, this SUV overestimation is only experienced in lesions within $15-20 \mathrm{~mm}$ from the bladder. This 


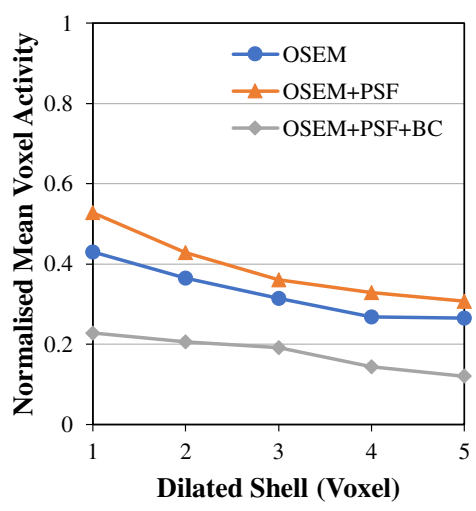

(a)

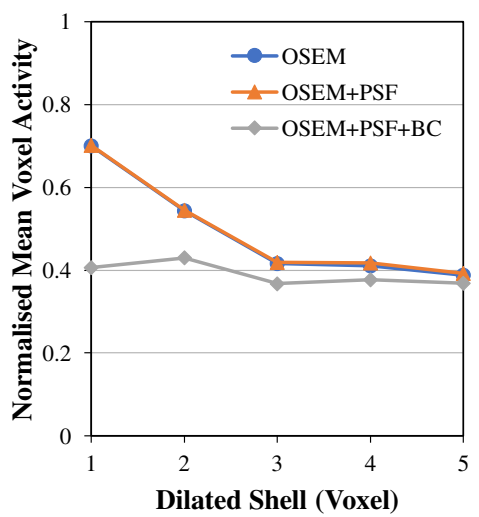

(c)

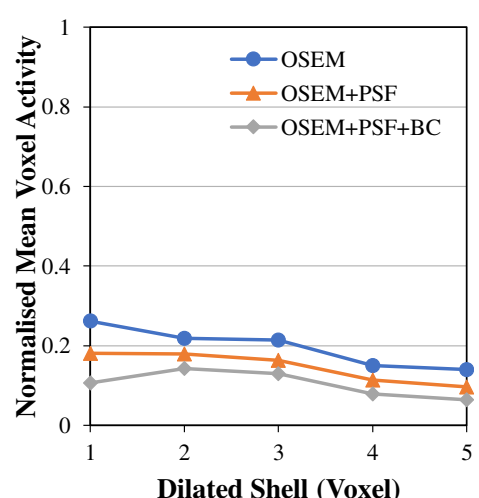

(b)

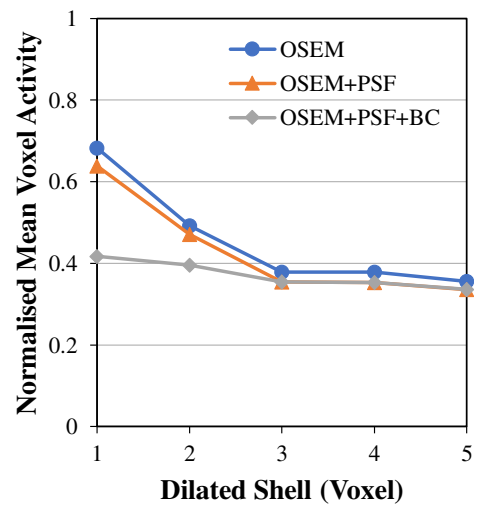

(d)

Fig. 12 Validating the effect and correction of spill-in effect using patient data. This figure shows the normalised mean values within the dilated shells surrounding the spleen at a 3 full iterations with no postfilter, b 20 full iterations with no post-filter, c 3 full iterations with a 4-mm Gaussian post-filter and $\mathbf{d} 20$ full iterations with a 4-mm Gaussian post-filter. The mean activity values in the dilated shells were normalised with the mean activity in the liver

improved result may be due to the improved resolution of the simulated GE PET/MR scanner, which makes the effect less prominent (as seen in Additional file 1: Figure S4).

We also discovered that the spill-in effect seems to increase with lesion size, and this is more evident in proximal lesions to the bladder (Fig. 5). As expected, spill-out effect from the lesions to the background causes an underestimation in lesion SUV for small diameter lesions, but the SUV increases as the diameter increases. However, the lesion SUV is further influenced by spill-in effect from the bladder to the lesions, and this is dependent on the lesion distance. Lesion L3 which is the closest lesion to the bladder has the highest bias in SUVs while the background lesion has the least. For lesion L3, the spill-in effect seems to increase with lesion diameter. This is because smaller lesions suffer from both spill-out effect to the colder region and spill-in effect from the hot background region. These two effects might sometimes cancel out, thereby giving a false impression of quantification accuracy in smaller lesions (as depicted in our study). However, for bigger lesions close to the bladder, spill-in effect is the major effect, thereby leading to a pronounced overestimation in SUV. For lesions L1 and L2, however, the impact of spill-in effect on SUV quantification is small, and only evident for 
$\mathrm{SUV}_{\max }$ (details in Additional file 1: Figure S2). This also re-iterates the strong dependence of spill-in effect on lesion distance.

It is worthy of note that the spill-in effect is more pronounced in lesion $\mathrm{SUV}_{\max }$ than $\mathrm{SUV}_{\text {mean }}$ as demonstrated by the results in Figs. 4, 5 and 6. This implies that if one is interested in quantification using $\mathrm{SUV}_{\text {mean }}$, then, the spill-in effect is minimal and can potentially be ignored. However, the fact that $\mathrm{SUV}_{\max }$ is the global clinical standard for quantification informs the need for proper spill-in correction. Our study also shows that SUV overestimation as a result of spill-in effect reduces over iteration (as seen in Figs. 6 and 12, and also Additional file 1: Figures S1, S5 and S6). Therefore, in order to reduce the spill-in effect, slightly increasing the number of iteration might be a good alternative. However, this will be at the expense of reduced CNR and increased noise.

$\mathrm{OSEM}+\mathrm{PSF}$ and $\mathrm{OSEM}+\mathrm{PSF}+\mathrm{BC}$ were used as correction techniques for the spill-in effect (their convergence properties are shown in Additional file 1: Figure S1). For L3, there does not seem to be any major improvement in OSEM+PSF reconstruction over OSEM especially for $\mathrm{SUV}_{\max }$ (as seen in Fig. 6). This could be because of the closeness of the lesion to the bladder, coupled with the blurring effect caused by post-filtering. This seems to nullify the recovery already obtained by incorporating PSF into the reconstruction algorithm, thereby making both ordinary OSEM and OSEM+PSF reconstructions to behave in a similar way. This blurring effect could further be confirmed by a pronounced bias at the bladder edges due to the spill-in activity from the bladder as shown in Fig. 7, as well as the edge analysis in Table 2. OSEM and OSEM+PSF are greatly affected by filtering, but OSEM+PSF+BC is more robust and less sensitive to filters. Another reason for the similar behaviour between OSEM and OSEM+PSF might be the simplistic spatially invariant resolution modelling in this study. This simple resolution modelling has been shown by past studies to increase edge artefact and system blurring [41].

From the NEMA phantom experiment (Fig. 11), this spill-in activity from the bottle can cause between 20 and 90\% activity overestimation in a sphere if it is within 1-2 voxels away from the hot bottle, but it reduces as we move further away from the bottle. The spill-in activity reduced greatly with OSEM+PSF+BC as it shows a value less than $3 \%$ in all cases. In this phantom experiment, spill-in effect is only prominent within 3 voxels (less than $10 \mathrm{~mm}$ ) around the hot bottle, whereas in the simulation study, this effect could potentially extend to about $15 \mathrm{~mm}$ with a very high activity in the bladder. This is an indication that this spill-in effect is strongly dependent on the activity in the hot region. Further validation of this technique using patient data also establishes the spill-in effect as a function of distance from the hot region (Fig. 12). OSEM and OSEM+PSF images show an increased activity value in the immediate vicinity of the spleen compared to $\mathrm{OSEM}+\mathrm{PSF}+\mathrm{BC}$ image, but this disparity in activity value decreases as we move further away from the spleen.

From the results shown in Figs. 6, 7 and 9, it could be seen that better lesion detection and quantification could be achieved with the recently proposed background correction technique, thereby potentially enhancing low contrast lesion detectability and better diagnosis. This was also confirmed by the NEMA phantom (Fig. 10) as OSEM $+\mathrm{PSF}+\mathrm{BC}$ demonstrate higher CNR than OSEM and OSEM+PSF especially at lower iterations and for smaller spheres. Also, the recently proposed background correction method has a stable performance for both lesion $\mathrm{SUV}_{\text {mean }}$ and $\mathrm{SUV}_{\max }$ (demonstrated 
by almost $0 \%$ change in Fig. 6) irrespective of the bladder activity and application of post-filter. This performance stability is an indication that the correction method can be used in the clinic for treatment response monitoring; however, this is still subject to further validation.

When compared with previously published results [8,27], our current study showed clearer and better results demonstrated on acquired PET data probably due to improved correction technique, incorporation of PSF in the reconstruction, and improved scanner resolution. There is an indication that the recently proposed background correction technique is robust and efficient in removing spill-in activity from the high-activity regions to the surroundings, thereby producing more reliable lesion quantification and better lesion visibility, compared with other correction techniques. Moreover, it is less tedious in that there is no need to calculate each lesion activity separately.

However, the recently proposed background correction technique is highly dependent on segmentation accuracy as shown in Fig. 8. This is important especially for pelvic scans where the bladder changes in volume and shape over time. Our result showed that the segmentation error is pronounced after 10 mins where the bladder expands majorly in an anterior direction towards the rectum and superior to the pelvic organs. This is in line with past studies $[42,43]$ which showed that bladder expands primarily in the superior anterior direction, and hence, addition of anisotropic margins to the bladder is necessary. The translation of this correction technique to clinical application would need to take into account the inaccuracy in bladder segmentation either by adding additional margins or by manual correction as suggested by past studies.

With the emergence of new tracers such as ${ }^{68} \mathrm{Ga}$-prostate-specific membrane antigen $\left({ }^{68} \mathrm{Ga}\right.$-PSMA) for prostate imaging, this correction technique can also be applicable for correcting shine-through effect in PET/MR imaging which causes loss of resolution and image artefacts due to its significant urinary excretion [19, 40]. Therefore, accurate correction will enhance reliable quantification of PET images and may lead to a tangible breakthrough in ${ }^{68} \mathrm{Ga}$-PSMA imaging.

\section{Limitations of the study}

A major limitation of this work is that there is no TOF implementation, whereas the modelled GE scanner supports TOF. Although TOF has been shown to mitigate errors due to data inconsistency [44], it is not yet certain if TOF implementation can sufficiently correct for spill-in effect, especially for proximal lesions (see TOF reconstructions from GE PET Toolbox in Additional file 1: Figures S5 and S6). However, work is currently ongoing on implementing this in the STIR library, and we therefore aim to include this in our future investigations. Another limitation is the lack of detailed realistic simulations to model some effects such as positron range, non-collinearity, detector response, inter-crystal scattering etc. Furthermore, a crucial approximation is that we modelled an image-based system blurring and used the same analytical model to simulate and reconstruct the data. Although this is helpful for investigating the performance of the algorithm, it would have been preferable to utilise Monte Carlo simulators. Nevertheless, these limitations do not cancel the relevance of the background correction method because we also utilised acquired phantom and patient datasets which demonstrated that the recently proposed background correction method successfully corrects for the background activity. 
Also, we used a spatially invariant model of the PSF in this work due to the complexities and computational demands associated with the use of a spatially variant model. Previous works have reported similar behaviour between the two models in terms of resolution, contrast and noise metrics $[41,45]$; however, we realise that a spatially variant model of the PSF is more accurate in reducing edge artefact and system blurring. We therefore aim to implement a realistic, spatially variant PSF modelling in our future work, as this might potentially help reduce both the spill-in and spill-out effects.

Clinical translation of this technique would need to place careful emphasis on the segmentation of the high-activity region. For example, it could be possible to use an MR image to segment the region as done in this study. Although segmentation could be performed on CT images as well, this is more challenging as PET/CT acquisitions are not performed simultaneously and there is the potential of bladder expansion between CT and PET scanning due to physiological motion. This has been reported to cause conspicuous distortions in lesions' shape and location. Another segmentation mismatch could also result from increase in bladder shape and size during a typical PET/MR scanning session. These issues could cause loss of resolution and inadequate quantification and also limit the applicability of this correction approach, as demonstrated in Fig. 8. A potential way of dealing with the segmentation inaccuracies might be to perform segmentation using multi-MR sequences, which could track the bladder change in shape and volume during a typical PET/MR scan. We therefore aim to incorporate appropriate corrections for bladder expansion and motion in our algorithm.

\section{Conclusion}

The effect of increasing activity from hot regions on adjacent lesion quantification, as well as the improvement brought about by the recently proposed background correction technique has been extensively studied in this work. This study shows that lesions relatively close to hot regions (within $15-20 \mathrm{~mm}$ ) are greatly affected by the spill-in effect, causing reduced visibility and activity overestimation of lesions. This effect is more pronounced in $\mathrm{SUV}_{\text {max }}$ than $\mathrm{SUV}_{\text {mean }}$ and reduces over iteration, but it is further aggravated by the use of filter. However, improved quantification and better lesion detectability were achieved with the recently proposed background correction technique irrespective of the lesion size, lesion distance from the hot region, the activity in the hot region or application of post-filter.

We could therefore conclude that the recently proposed background correction method is appropriate for reliable quantification and diagnosis of lesions near a hot region. This is particularly important when examining the pelvic areas for infection, metastases and cancer. Furthermore, this correction technique is not limited to pelvic imaging. It could potentially be applied to imaging of any high-activity region such as the brain, head and neck, myocardium, and bone.

\section{Additional file}

Additional file 1: Figure S1. Convergence plots of all the reconstruction algorithms using lesion SUV $\max$ and SUV $V_{\text {mean. }}$. Figure S2. The variation of the lesion uptake with lesion size and bladder SUV. Figure S3. The dependence of spill-in effect on post-filter. This was done with a single noise realisation at 30 iterations. Figure S4. The spill-in activity from the bladder to the surroundings as a function of the system resolution (in FWHM), using Eq. 2. Figure S5. The normalised mean values within the dilated shells surrounding the NEMA bottle obtained at with (a) 3 iterations and (b) 20 iterations, with non-TOF and TOF 
reconstructions. Figure S6. Normalised mean values within the dilated shells surrounding the spleen obtained with non-TOF and TOF reconstructions at (a) 3 iterations and (b) 20 iterations. Table S1. This shows how bladder SUV affects lesion quantification for each lesion diameter. This is expressed as the \% change in lesion SUV as bladder SUV increases. The SUV values are the mean values from all noise realisations at 30 full iterations with $4 \mathrm{~mm}$ post-filter. (DOCX $139 \mathrm{~kb})$

\section{Abbreviations}

${ }^{68} \mathrm{Ga}$-PSMA: Prostate-specific membrane antigen labelled with ${ }^{68} \mathrm{Ga}$; BC: Background Correction; CNR: Contrast-to-noise ratio; FOV: Field of view; FWHM: Full width at half maximum; GE: General Electric; IQ: Image quality; OSEM: Ordered subset expectation maximisation; PET: Positron emission tomography; PSF: Point-spread function; RD: Ring difference; ROI: Region of interest; STIR: Software for Tomographic Image Reconstruction; SUV: Standardised uptake values

\section{Acknowledgements}

We would like to acknowledge Dr. Steven Sourbron, Mr. Daniel Deidda (University of Leeds), Dr. Irene Polycarpou (European University of Cyprus), Nicholas Keat (Invicro), Professor Stefaan Vandenberghe and Esther D'Hoe (University of Ghent) for their support during the study. Thanks to Miss Richa Gandhi, Mr. Waqas Ahmad and Mr. Saul Jones for proof-reading this manuscript.

This work was undertaken on MARC1, part of the High Performance Computing and Leeds Institute for Data Analytics (LIDA) facilities at the University of Leeds, UK.

We are also thankful to Dr. Kris Thielemans (UCL) and several scientists at GE Healthcare (Michel Tohme, Floris Jansen Gaspar Delso) for helping us to reconstruct the PET data from the GE Signa PET scanner.

\section{Funding}

Mrs. Mercy Akerele is supported through a PhD research scholarship by the Schlumberger Faculty for the Future Miss Palak Wadhwa is supported by an MRC CASE PhD scholarship (MR/M01746X/1). Dr. Charalampos Tsoumpas is supported by a Royal Society industry research fellowship (IF170011).

\section{Authors' contributions}

MIA designed the study, performed data simulation and reconstructions, analysed the results and wrote the manuscript. PW performed the phantom experiment, reconstructed the phantom and patient data with GE Toolbox and also helped towards reading and reconstructing the GE data using STIR software. JS developed the background correction method and also contributed some ideas towards the study. WH helped with the phantom and patient data acquisition. CT conceived the original idea, supervised the study and helped with restructuring of the manuscript and the revision process.

All authors read and approved the final manuscript.

\section{Ethics approval and consent to participate}

The data was generated under the ethics number 17/WM/0084 with permission from a clinical study performed at Invicro.

\section{Consent for publication}

Informed consent was obtained from all individual participants included in the study, including the publication of results.

\section{Competing interests}

The authors declare that they have no competing interests.

\section{Publisher's Note}

Springer Nature remains neutral with regard to jurisdictional claims in published maps and institutional affiliations.

\section{Author details}

${ }^{1}$ Biomedical Imaging Science Department, School of Medicine, University of Leeds, Leeds, West Yorkshire, UK. ${ }^{2}$ Invicro, Hammersmith Hospital, London, UK. ${ }^{3}$ Molecular Imaging Research Group, Health Research Institute of Santiago de Compostela (IDIS), Santiago de Compostela, Galicia, Spain.

Received: 25 April 2018 Accepted: 20 November 2018

Published online: 05 December 2018

\section{References}

1. Hoh CK. Clinical use of FDG PET. Nucl Med Biol. 2007;34(7):737-42.

2. Ben-Haim S, Ell P. ${ }^{18}$ F-FDG PET and PET/CT in the evaluation of cancer treatment response. J Nucl Med. 2009:50(1):88-99.

3. Capirci C, Rampin L, Erba PA, Galeotti F, Crepaldi G, Banti E, et al. Sequential FDG-PET/CT reliably predicts response of locally advanced rectal cancer to neo-adjuvant chemo-radiation therapy. Eur J Nucl Med Mol Imaging. 2007;34(10): 1583-93.

4. Nahmias C, Wahl LM. Reproducibility of standardized uptake value measurements determined by 18 F-FDG PET in malignant tumors. J Nucl Med. 2008;49(11):1804-8.

5. Soret M. Partial volume effect in PET tumor imaging. J Nucl Med. 2007;48(6):932-45.

6. Du Y, Madar I, Stumpf MJ, Rong X, Fung GSK, Frey EC. Compensation for spill-in and spill-out partial volume effects in cardiac PET imaging. J Nucl Cardiol. 2013;20(1):84-98. 
7. Forsythe RO, Dweck MR, McBride OMB, Vesey AT, Semple SI, Shah ASV, et al. ${ }^{18} \mathrm{~F}$-sodium fluoride uptake in abdominal aortic aneurysms: the SoFIA Study. J Am Coll Cardiol. 2018;71(5):513-23.

8. Liu Y. Invalidity of SUV measurements of lesions in close proximity to hot sources due to 'shine-through' effect on FDG PET-CT interpretation. Radiol Res Pract. 2012;2012:867218.

9. Gaertner FC, Beer AJ, Souvatzoglou M, Eiber M, Furst S, Ziegler SI, et al. Evaluation of feasibility and image quality of 68Ga-DOTATOC positron emission tomography/magnetic resonance in comparison with positron emission tomography/computed tomography in patients with neuroendocrine tumors. Investig Radiol. 2013;48(5):263-72.

10. Afshar-Oromieh A, Haberkorn U, Schlemmer HP, Fenchel M, Eder M, Eisenhut M, et al. Comparison of PET/CT and PET/ MRI hybrid systems using a 68Ga-labelled PSMA ligand for the diagnosis of recurrent prostate cancer: initial experience. Eur J Nucl Med Mol Imaging. 2014;41(5):887-97.

11. Koyama K, Okamura T, Kawabe J, Ozawa N, Torii K, Umesaki N, et al. Evaluation of 18F-FDG PET with bladder irrigation in patients with uterine and ovarian tumors. J Nucl Med. 2003;44:353-8.

12. Moran JK, Lee HB, Blaufox MD. Optimization of urinary FDG excretion during PET imaging. J Nucl Med. 1999;40:1352-7.

13. Kosuda S, Fisher S, Wahl RL. Animal studies on the reduction and/or dilution of 2-deoxy-2-18F fluoro-D-glucose (FDG) activity in the urinary system. Ann Nucl Med. 1997;11(3):213-8.

14. Bach-Gansmo T, Dybvik JA, Adamsen TC, Naum A. Variation in urinary excretion of FDG, yet another uncertainty in quantitative PET. Acta Radiol Short Rep. 2012;1 (8):1-3.

15. Lo E, Nicolle LE, Coffin SE, Gould C, Maragakis LL, Meddings J, et al. Strategies to prevent catheter-associated urinary tract infections in acute care hospitals: 2014 update. Infect Control Hosp Epidemiol. 2014;35(5):464-79.

16. Beheshti M, Haim S, Zakavi R, Steinmair M, Waldenberger P, Kunit T, et al. Impact of ${ }^{18} \mathrm{~F}$-choline PET/CT in prostate cancer patients with biochemical recurrence: influence of androgen deprivation therapy and correlation with PSA kinetics. J Nucl Med. 2013;54:833-40.

17. Rauscher I, Maurer T, Fendler WP, Sommer WH, Schwaiger M, Eiber M. ${ }^{68} \mathrm{Ga}-\mathrm{PSM} A$ ligand PET/CT in patients with prostate cancer: how we review and report. Cancer Imaging. 2016;16:14.

18. Witney T, Alam I, Turton D. Evaluation of deuterated 18F-and 11C-labeled choline analogs for cancer detection by positron emission tomography. Clin Cancer Res. 2012;18(4):1063-72.

19. Steiner C, Vees H, Zaidi H, Wissmeyer M, Berrebi O, Kossovsky MP, et al. Three-phase 18F-fluorocholine PET/CT in the evaluation of prostate cancer recurrence. Nuklearmedizin. 2009:48:1-9.

20. Thomas BA, Erlandsson K, Modat M, Thurfjell L, Vandenberghe R, Ourselin S, et al. The importance of appropriate partial volume correction for PET quantification in Alzheimer's disease. Eur J Nucl Med Mol Imaging. 2011;38(6):1104-19.

21. Hofheinz F, Langner J, Petr J, Beuthien-Baumann B, Oehme L, Steinbach J, et al. A method for model-free partial volume correction in oncological PET. EJNMMI Res. 2012;2:16.

22. Munk OL, Tolbod LP, Hansen SB, Bogsrud TV. Point-spread function reconstructed PET images of sub-centimeter lesions are not quantitative. EJNMMI Phys. 2017:4:5.

23. Cysouw MCF, Kramer GM, Schoonmade LJ, Boellaard R, de Vet HCW, Hoekstra OS. Impact of partial volume correction in oncological PET studies: a systematic review and meta-analysis. Eur J Nucl Med Mol Imaging. 2017:44(12):2105-16.

24. Frouin V, Comtat C, Reilhac A, Gregoire MC. Correction of partial-volume effect for PET striatal imaging: fast implementation and study of robustness. J Nucl Med. 2002;43(12):1715-26.

25. Geworski L, Knoop BO, de Cabrejas ML, Knapp WH, Munz DL. Recovery correction for quantitation in emission tomography: a feasibility study. Eur J Nucl Med. 2000;27(2):161-9.

26. Cal-Gonzalez J, Li X, Heber D, Rausch I, Moore SC, Schäfers K, et al. Partial volume correction for improved PET quantification in 18F-NaF imaging of atherosclerotic plaques. J Nucl Cardiol. 2018;25(5):1742-56.

27. Silva-Rodriguez J, Tsoumpas C, Dominguez-Prado I, Pardo-Montero J, Ruibal A, Aguiar P. Impact and correction of the bladder uptake on 18F-FCH PET quantification: a simulation study using the XCAT2 phantom. Phys Med Biol. 2016;61(2): 758-73.

28. Segars W, Sturgeon G, Mendonca S, Grimes J, Tsui B. 4D XCAT phantom for multimodality imaging research. Med Phys. 2010;37(9):4902-15.

29. Ziegler S, Jakoby BW, Braun H, Paulus DH, Quick HH. NEMA image quality phantom measurements and attenuation correction in integrated PET/MR hybrid imaging. EJNMMI Physics. 2015;2:18.

30. Grant AM, Deller TW, Khalighi MM, Maramraju SH, Delso G, Levin CS. NEMA NU 2-2012 performance studies for the SiPM-based ToF-PET component of the GE SIGNA PET/MR system. Med Phys. 2016;43(5):2334.

31. Pelosi E, Skanjeti A, Scapoli P. Improved diagnosis of pelvic lesions with dual-phase (18F)FDG-PET/CT. Radiol Case Rep. 2011;6(1):149.

32. Park SH, Kim M-J, Kim JH, Cho AE-H, Park M-S, Kim KW. FDG uptake in PET by bladder hernia simulating inguinal metastasis. Yonsei Med J. 2007:48(5):886-90.

33. Puri T, Greenhalgh TA, Wilson JM, Franklin J, Wang LM, Strauss V, et al. $\left[{ }^{18} \mathrm{~F}\right]$ Fluoromisonidazole PET in rectal cancer. EJNMMI Res. 2017:7:78

34. Lee SW, Kim JH. The significance of natural bladder filling by the production of urine during cystometry. Neurourol Urodyn. 2008;27(8):772-4.

35. Thielemans K, Tsoumpas C, Mustafovic S, Beisel T, Aguiar P, Dikaios N, et al. STIR: software for tomographic image reconstruction release 2. Phys Med Biol. 2012;57(4):867-83.

36. Levin CS, Maramraju H, Khalighi MM, Deller TW, Delso G, Jansen F. Design features and mutual compatibility studies of the time-of-flight PET capable GE SIGNA PET/MRI system. IEEE Trans Med Imag. 2016;35(8):1907-14.

37. Siddon RL. Fast calculation of the exact radiological path for a three-dimensional CT array. Med Phys. 1985:12:252-5.

38. Rapisarda E, Bettinardi V, Thielemans K, Gilardi MC. Image-based point spread function implementation in a fully 3D OSEM reconstruction algorithm for PET. Phys Med Biol. 2010;55(14):4131-51.

39. Rogasch JM, Hofheinz F, Lougovski A, et al. The influence of different signal-to-background ratios on spatial resolution and F18-FDG-PET quantification using point spread function and time-of-flight reconstruction. EJNMMI Phys. 2014;1 (1):12.

40. Kolb A, Sauter AW, Eriksson L, Vandenbrouke A, Liu CC, Levin C, et al. Shine-through in PET/MR imaging: effects of the magnetic field on positron range and subsequent image artifacts. J Nucl Med. 2015;56(6):951-4.

41. Alessio AM, Stearns CW, Tong S, Ross SG, Kohlmyer S, Ganin A, et al. Application and evaluation of a measured spatially variant system model for PET image reconstruction. IEEE Trans Med Imag. 2010;29(3):938-49. 
42. Foroudi F, Pham D, Bressel M, Gill S, Kron T. Intrafraction bladder motion in radiation therapy estimated from pretreatment and posttreatment volumetric imaging. Int J Radiat Oncol Biol Phys. 2013;86(1):77-82.

43. Grønborg C, Vestergaard A, Høyer M, Söhn M, Pedersen EM, Petersen JB, et al. Intra-fractional bladder motion and margins in adaptive radiotherapy for urinary bladder cancer. Acta Oncol. 2015;54(9):1461-6.

44. Vandenberghe S, Mikhaylova E, D'Hoe E, Mollet P, Karp JS. Recent developments in time-of-flight PET. EJNMMMI Phys. 2016;3:3.

45. Teo BK, Seo Y, Bacharach SL, Carrasquillo JA, Libutti SK, Shukla H, et al. Partial volume correction in PET: validation of an iterative postreconstruction method with phantom and patient data. J Nucl Med. 2007:48:802-10.

Submit your manuscript to a SpringerOpen ${ }^{\circ}$ journal and benefit from:

- Convenient online submission

- Rigorous peer review

- Open access: articles freely available online

- High visibility within the field

- Retaining the copyright to your article 